\title{
Inflammation and Nutritional Risk: A Feature to Consider in Planned Oncologic Surgery
}

\author{
Alessio Molfino - Irma Kushta - Filippo Rossi Fanelli • \\ Maurizio Muscaritoli
}

Published online: 2 August 2009

(C) Société Internationale de Chirurgie 2009

\section{Introduction}

Deterioration of nutritional status is associated with metabolic changes, organ failure, and immune status impairment, as confirmed by the recent study by Antoun et al. [1]. This study highlights the major role of blood albumin levels $<30 \mathrm{~g} / \mathrm{l}$ as a major risk factor for complications in surgical cancer patients, whereas a preoperative involuntary weight loss as high as $15 \%$ is related to the onset of major postoperative complications [1]. There is now consistent evidence that the presence of systemic inflammation is associated with increased weight loss and, in particular, with loss of lean body mass and functional decline [2]. The basis of the systemic inflammatory response in cancer is still strongly debated. Alterations in neuroendocrine metabolism, humoral changes, including elevated interleukins, interferons, and acute phase proteins levels, have been described [3]. Hepatocytes play a major role in the elaboration of the systemic inflammatory response, as they are implicated in the synthesis and release into plasma of several acute phase proteins, such as C-reactive protein (CRP), which sustains the inflammatory response while negatively affecting albumin synthesis. CRP represents the most commonly used serum marker to investigate systemic inflammation in patients with cancer due to its sensitivity, specificity, and high reproducibility [2,4]. An interesting point is that as CRP levels increase, albumin decreases; this observation is detectable among different tumor types [2]. Therefore, in the study by Antoun et al. the evaluation of CRP levels should have been strongly considered to better assess nutritional

A. Molfino - I. Kushta · F. Rossi Fanelli - M. Muscaritoli ( $\)$ Department of Clinical Medicine, Sapienza University of Rome, Viale dell'Università 37, 00185 Rome, Italy

e-mail: maurizio.muscaritoli@uniroma1.it

risk. In fact, cancer patients with albumin levels $<30 \mathrm{~g} / \mathrm{l}$ might maintain an enhanced systemic inflammatory response as expressed by increased CRP levels.

Recent evidences show that genetic predisposition of the host to local or systemic inflammation may contribute to cancer cachexia [5]. In fact, interleukin-10 genotype of the host can favor the development of severe protein-energy malnutrition among patients with gastroesophageal malignancies. Interestingly, in the same population, increased weight loss was highly correlated with CRP levels [5].

Taken together, these data suggest that clinicians should consider evaluating CRP in planned oncologic surgery patients in association with the other clinical and biological parameters indicated by Antoun et al. [1]. Systemic inflammatory response should be routinely assessed in patients with cancer, not only to treat but also to prevent cancer cachexia-related multiple complications, thereby reducing morbidity, mortality, and improving quality of life.

\section{References}

1. Antoun S, Rey A, Béal J et al (2009) Nutritional risk factors in planned oncologic surgery: what clinical and biological parameters should be routinely used? World J Surg 33:1633-1640

2. McMilan DC (2008) An inflammation-based prognostic score and its role in the nutrition-based management of patients with cancer. Proc Nutr Soc 67:257-262

3. Gabay C, Kushner I (1999) Acute-phase proteins and other systemic responses to inflammation. N Engl J Med 340:448-454

4. Marsic C, Kazemi-Shirazi L, Schichbauer T et al (2008) C-reactive protein and all-cause mortality in a large-based cohort. Clin Chem 54:343-349

5. Deans DA, Tan BH, Ross JA et al (2009) Cancer cachexia is associated with the IL10-1082 gene promoter polymorphism in patients with gastroesophageal malignancy. Am J Clin Nutr 89:1164-1172 